\title{
Rotational Dynamics of Proteins from Spin Relaxation Times and Molecular Dynamics Simulations
}

\author{
O. H. Samuli Ollila, ${ }^{*} \dagger+₫$ Harri A. Heikkinen, ${ }^{\dagger}$ and Hideo Iwai ${ }^{\dagger} \odot$
}

${ }^{\dagger}$ Research Program in Structural Biology and Biophysics, Institute of Biotechnology, University of Helsinki, 00014 Helsinki, Finland

${ }^{\ddagger}$ Institute of Organic Chemistry and Biochemistry, Czech Academy of Sciences, 11720 Prague 6, Czech Republic

Supporting Information

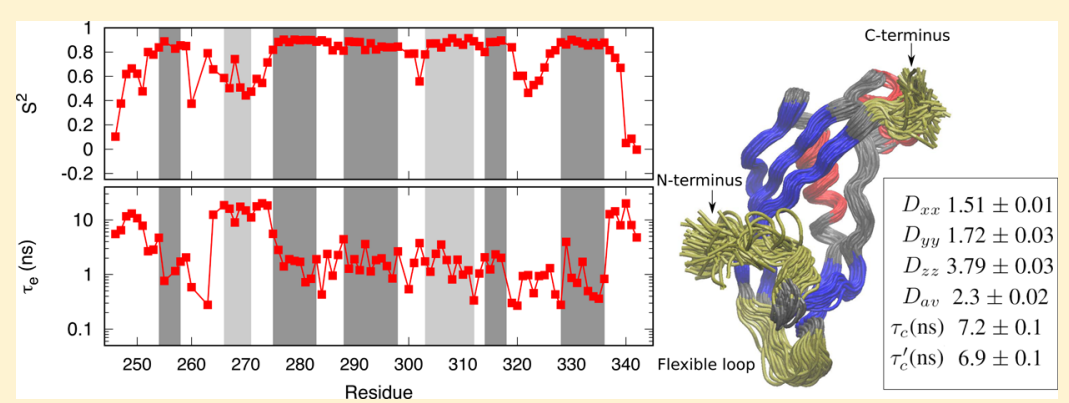

ABSTRACT: Conformational fluctuations and rotational tumbling of proteins can be experimentally accessed with nuclear spin relaxation experiments. However, interpretation of molecular dynamics from the experimental data is often complicated, especially for molecules with anisotropic shape. Here, we apply classical molecular dynamics simulations to interpret the conformational fluctuations and rotational tumbling of proteins with arbitrarily anisotropic shape. The direct calculation of spin relaxation times from simulation data did not reproduce the experimental data. This was successfully corrected by scaling the overall rotational diffusion coefficients around the protein inertia axes with a constant factor. The achieved good agreement with experiments allowed the interpretation of the internal and overall dynamics of proteins with significantly anisotropic shape. The overall rotational diffusion was found to be Brownian, having only a short subdiffusive region below 0.12 ns. The presented methodology can be applied to interpret rotational dynamics and conformation fluctuations of proteins with arbitrary anisotropic shape. However, a water model with more realistic dynamical properties is probably required for intrinsically disordered proteins.

\section{INTRODUCTION}

Conformational fluctuations and the entropy of proteins play a significant role in their functionality and interactions with other biomolecules. Conformational fluctuations and the overall Brownian tumbling of proteins are experimentally accessible through the spin relaxation times of ${ }^{15} \mathrm{~N}$ and ${ }^{13} \mathrm{C}$ nuclei measured with nuclear magnetic resonance (NMR) techniques. $^{1-7}$ The spin relaxation rates have been used to, for example, analyze conformational entropies, ${ }^{1,8-11}$ binding entropies, ${ }^{1,12}$ resolve sampled structures, ${ }^{3-5,13}$ and validate molecular dynamics (MD) simulations. ${ }^{14-21}$ These analyses are almost exclusively based on the separation of the internal conformational fluctuations and the overall rotational tumbling. ${ }^{22,23}$ Also, the isotropic overall diffusion is often assumed, whereas analysis of anisotropic molecules is significantly more complicated. ${ }^{1,24-27}$ Thus, new approaches are needed to interpret spin relaxation times measured from anisotropic or intrinsically disordered molecules.

Classical MD simulation methods are promising tools to interpret spin relaxation experiments for molecules with significantly anisotropic shape or correlations between internal and overall rotational motions. Practical applications are, however, limited by inaccuracies in the force field descriptions and the available time scales in the simulations. ${ }^{17-19,28-31}$ The main issues have been the overestimated overall rotational diffusion of proteins due to inaccuracies in water models ${ }^{19,29,32}$ and the insufficient accuracy of correlation functions calculated from single molecules in MD simulations. ${ }^{30,33}$

In this work, we overcome these issues by assuming that the overall rotational dynamics of protein follows anisotropic rigid body diffusion. Diffusion coefficients around inertia axes are directly calculated from angular displacements. The diffusion coefficients are then used to determine the contribution of the overall rotational tumbling to the rotational correlation functions of $\mathrm{N}-\mathrm{H}$ bonds in the protein backbone. This reduces the required simulation length for the accurate determination of the rotational correlation functions. Furthermore, the overestimated overall Brownian tumbling rates due to the inaccurate water model can be corrected during the correlation function calculation by scaling the diffusion coefficients in all directions with a constant factor. The corrected correlation functions can be used to interpret the

Received: March 7, 2018

Revised: $\quad$ May 27, 2018

Published: May 29, 2018 
spin relaxation experiments for proteins with arbitrarily anisotropic shapes.

The developed approach is demonstrated by interpreting the experimental spin relaxation data of C-terminal domains of TonB proteins from Helicobacter pyroli (HpTonB-92) $)^{34}$ and from Pseudomonas aeruginosa (PaTonB-96), ${ }^{35}$ having 92 and 96 residues, respectively. Both proteins have significantly anisotropic shape, which would complicate the standard spin relaxation data analysis. ${ }^{1,2,24-27}$

\section{METHODS}

Spin Relaxation Experiments and Rotational Dynamics of Molecules. Molecular dynamics of the protein backbone residues and spin relaxation experiments can be connected by using the spectral density $J(\omega)$

$$
J(\omega)=2 \int_{0}^{\infty} C(t) \cos (\omega t) \mathrm{d} t
$$

which is the Fourier transformation of the second-order rotational correlation function for $\mathrm{N}-\mathrm{H}$ bond vector

$$
C(t)=\left\langle\frac{3}{2} \cos ^{2} \theta_{t^{\prime}+t}-\frac{1}{2}\right\rangle_{t^{\prime}}
$$

where $\theta_{t^{\prime}+t}$ is the $\mathrm{N}-\mathrm{H}$ bond angle between times $t^{\prime}$ and $t^{\prime}+t$ and angular brackets refer to the ensemble average. Connection to the experimentally measured spin relaxation times $T_{1}, T_{2}$ and the nuclear Overhauser effect (NOE) relaxation is given by the Redfield equations ${ }^{36,37}$

$$
\begin{aligned}
\frac{1}{T_{1}}= & \frac{d_{\mathrm{NH}}^{2} N_{\mathrm{H}}}{20}\left[J\left(\omega_{\mathrm{H}}-\omega_{\mathrm{N}}\right)+3 J\left(\omega_{\mathrm{N}}\right)+6 J\left(\omega_{\mathrm{N}}+\omega_{\mathrm{H}}\right)\right] \\
& +\frac{\left(\sigma \omega_{\mathrm{N}}\right)^{2}}{15} J\left(\omega_{\mathrm{N}}\right) \\
\frac{1}{T_{2}}= & \frac{1}{2} \frac{d_{\mathrm{NH}}^{2} N_{\mathrm{H}}}{20}\left[4 J(0)+3 J\left(\omega_{\mathrm{N}}\right)+J\left(\omega_{\mathrm{H}}-\omega_{\mathrm{N}}\right)+6 J\left(\omega_{\mathrm{H}}\right)\right. \\
+ & \left.6 J\left(\omega_{\mathrm{N}}+\omega_{\mathrm{H}}\right)\right]+\frac{\left(\sigma \omega_{\mathrm{N}}\right)^{2}}{90}\left[4 J(0)+3 J\left(\omega_{\mathrm{N}}\right)\right] \\
\mathrm{NOE} & =1+\frac{d_{\mathrm{NH}}^{2} N_{\mathrm{H}}}{20}\left[6 J\left(\omega_{\mathrm{N}}+\omega_{\mathrm{H}}\right)+J\left(\omega_{\mathrm{H}}-\omega_{\mathrm{N}}\right)\right] \frac{\gamma_{\mathrm{H}} T_{1}}{\gamma_{\mathrm{N}}}
\end{aligned}
$$

where $\omega_{\mathrm{N}}$ and $\omega_{\mathrm{H}}$ are the Larmor angular frequencies of ${ }^{15} \mathrm{~N}$ and ${ }^{1} \mathrm{H}$, respectively, and the number of bound protons $N_{\mathrm{H}}=1$ for $\mathrm{N}-\mathrm{H}$ bonds. The dipolar coupling constant is given by

$$
d_{\mathrm{NH}}=-\frac{\mu_{0} \hbar \gamma_{\mathrm{H}} \gamma_{\mathrm{N}}}{4 \pi\left\langle r_{\mathrm{NH}}{ }^{3}\right\rangle}
$$

where $\mu_{0}$ is the magnetic constant or vacuum permeability, $\hbar$ is the reduced Planck constant, and $\gamma_{\mathrm{N}}$ and $\gamma_{\mathrm{H}}$ are the gyromagnetic constants of ${ }^{15} \mathrm{~N}$ and ${ }^{1} \mathrm{H}$, respectively. The average cubic length is calculated as $\left\langle r_{\mathrm{NH}}{ }^{3}\right\rangle=(0.101 \mathrm{~nm})$, and the value of $\Delta \sigma=-160 \mathrm{ppm}$ is used for the chemical shift anisotropy of $\mathrm{N}-\mathrm{H}$ bonds in proteins. ${ }^{37,38}$

Spin relaxation experiments are typically interpreted for proteins by assuming that the motions related to the overall Brownian tumbling and conformational fluctuations are independent. $^{39}$ The rotational correlation function for each $\mathrm{N}-\mathrm{H}$ bond can be then written as ${ }^{1,2,22,23,39}$

$$
C(t)=C_{\mathrm{I}}(t) C_{\mathrm{O}}(t)
$$

where $C_{\mathrm{I}}(t)$ and $C_{\mathrm{O}}(t)$ are correlation functions for the internal dynamics and overall rotations, respectively. Conformational fluctuations can be described in this approximation by using the square of the order parameter with respect to molecular axes $S^{2}$, which are given by the plateau of the internal rotational correlation function. Timescales for the fluctuations can be characterized by using the effective correlation time

$$
\tau_{\text {eff }}=\int_{0}^{\infty} C_{\mathrm{I}}^{\prime}(t) \mathrm{d} t
$$

where $C_{\mathrm{I}}^{\prime}(t)=\frac{C(t)_{\mathrm{I}}-S^{2}}{1-S^{2}}$ is the reduced correlation function. ${ }^{23}$

The overall rotational correlation function is often described by approximating the protein as a rigid body. For arbitrarily anisotropic molecules, the correlation functions can be presented as a sum of five exponentials ${ }^{2,24}$

$$
C_{\mathrm{O}}(t)=\sum_{j=1}^{5} A_{j} \mathrm{e}^{-t / \tau_{j}}
$$

where the prefactors $A_{j}$ depend on the directions of chemical bonds with respect to the molecular axes ${ }^{24,26}$ and the time constants $\tau_{j}$ are related to the diffusion constants around three principal axes of a molecule $\left(D_{x}, D_{y}\right.$, and $\left.D_{z}\right)$ through equations $s^{2,24}$

$$
\begin{aligned}
\tau_{1} & =\left(4 D_{x}+D_{y}+D_{z}\right)^{-1} \\
\tau_{2} & =\left(D_{x}+4 D_{y}+D_{z}\right)^{-1} \\
\tau_{3} & =\left(D_{x}+D_{y}+4 D_{z}\right)^{-1} \\
\tau_{4} & =\left[6\left(D_{\mathrm{av}}+\left(D_{\mathrm{av}}{ }^{2}-L^{2}\right)^{1 / 2}\right)\right]^{-1} \\
\tau_{5} & =\left[6\left(D_{\mathrm{av}}-\left(D_{\mathrm{av}}{ }^{2}-L^{2}\right)^{1 / 2}\right)\right]^{-1}
\end{aligned}
$$

where

$$
D_{\mathrm{av}}=\frac{1}{3}\left(D_{x}+D_{y}+D_{z}\right) \text { and } L^{2}=\frac{1}{3}\left(D_{x} D_{y}+D_{x} D_{z}+D_{y} D_{z}\right)
$$

The simplest approach to extract molecular dynamics from the experimental data is the original "model-free analysis", 23 where an isotropic diffusion is assumed for the overall rotation of the protein. This reduces eq 8 to a monoexponential form and the overall rotational dynamics can be described with a single time constant $\tau_{\mathrm{c}}$. Also, the internal correlation functions for each residue are assumed to decay exponentially with a single time constant $\tau_{\text {eff }}$ toward to the square of the order parameter $S^{2}$. The three parameters $\left(\tau_{\mathcal{c}} \tau_{\text {eff }}\right.$ and $\left.S^{2}\right)$ can be then successfully resolved from a fit to the experimental data. However, the number of parameters to be fitted increases if the protein experiences an anisotropic overall diffusion or has several timescales for internal motions. In this case, the fitting becomes often ambiguous, even if the experimental data would be measured with multiple magnetic field strengths. ${ }^{1,26,40}$ The anisotropic rotational diffusion is sometimes described with hydrodynamical calculations but they are sensitive to the estimation of the hydration shell around the protein. ${ }^{41}$

A rough estimate for the timescale of overall rotational dynamics is often given by using the $T_{1} / T_{2}$ ratio. ${ }^{37}$ This is based on the assumptions that $T_{1}$ and $T_{2}$ are independent of the internal motions and that the overall dynamics is isotropic. The spectral density then reduces to 


$$
J^{\prime}(\omega)=S^{2} \frac{\tau_{\mathrm{c}}^{\prime}}{1+\left(\omega \tau_{\mathrm{c}}^{\prime}\right)^{2}}
$$

and the correlation time describing the overall rotational motion, $\tau_{\mathcal{c}}^{\prime}$, can be estimated by numerically minimizing the equation

$$
\frac{T_{1}}{T_{2}} \approx \frac{\frac{1}{2} \frac{d_{\mathrm{NH}}^{2} N_{\mathrm{H}}}{20}\left[4 J^{\prime}(0)+3 J^{\prime}\left(\omega_{\mathrm{N}}\right)+J^{\prime}\left(\omega_{\mathrm{H}}-\omega_{\mathrm{N}}\right)+6 J^{\prime}\left(\omega_{\mathrm{H}}\right)+6 J^{\prime}\left(\omega_{\mathrm{N}}+\omega_{\mathrm{H}}\right)\right]+\frac{\left(\sigma \omega_{\mathrm{N}}\right)^{2}}{90}\left[4 J^{\prime}(0)+3 J^{\prime}\left(\omega_{\mathrm{N}}\right)\right]}{\frac{d_{\mathrm{NH}}^{2} N_{\mathrm{H}}}{20}\left[J^{\prime}\left(\omega_{\mathrm{H}}-\omega_{\mathrm{N}}\right)+3 J^{\prime}\left(\omega_{\mathrm{N}}\right)+6 J^{\prime}\left(\omega_{\mathrm{N}}+\omega_{\mathrm{H}}\right)\right]+\frac{\left(\sigma \omega_{\mathrm{N}}\right)^{2}}{15} J^{\prime}\left(\omega_{\mathrm{N}}\right)}
$$

with respect to the experimentally measured $T_{1} / T_{2}$ ratio.

Rotational Dynamics from MD Simulations. A classical MD simulation gives a trajectory for each atom in the system as a function of time. Rotational correlation functions for each bond can be then directly calculated from the trajectories by eq 2 and used to calculate the spin relaxation times through eqs $1-5$. The resulting values can be compared to experimental data to assess simulation model quality ${ }^{14-21,31,42}$ and to interpret experiments. ${ }^{21,42,43}$

The direct comparison with experiments is, however, often complicated by the insufficient statistics for the calculated correlation functions and the overestimated rotational diffusion due to inaccuracies in the used water models. ${ }^{29,30,32}$ Here, we show that the statistical accuracy of the contribution of the overall tumbling to the correlation functions, $C_{\mathrm{O}}(t)$, in eq 6 , can be increased for rigid proteins by directly calculating the diffusion coefficients of the inertia axes. The rotational diffusion coefficients can be related to the timescales $\tau_{j}$ of the correlation function for anisotropic rigid body rotation in eq 8 by using the relations in eq $9 .^{24}$

The rotational diffusion coefficients are calculated by fitting a linear slope to the square angle deviation of the inertia axes (see below). Lag times up to one hundredth of the total simulation length were used. This is expected to be the maximum lag time for the good statistics of rotational dynamics analyzed from a single molecule in $\mathrm{MD}$ simulations. ${ }^{33}$ Error bars for the diffusion coefficients were defined to include results when the lag time was varied with \pm 1 ns. This requires less simulation data for the good statistics than a direct fit of the multiexponential sum in eq 8 to the rotational correlation function calculated from MD simulation. In addition, the overestimated rotational diffusion due to the water model ${ }^{19,29,32}$ can be corrected by scaling the diffusion coefficients around all inertia axes by a constant factor. This approach takes into account the anisotropic shape of the molecule. This is a significant advancement to the previous studies, which assume isotropic rotational diffusion with a single exponential rotational correlation function ${ }^{10,15-17,44}$ or use order parameters to compare simulations with experimental data. ${ }^{14,17,18,44}$

The practical analysis can be divided into seven steps as follows:

(1) The total rotational correlation functions $C(t)$ for $\mathrm{N}-\mathrm{H}$ bond vectors in a protein are directly calculated from the MD simulation trajectory by applying eq 2 .

(2) The rotational correlation functions for internal dynamics $C_{\mathrm{I}}(t)$ are calculated from the MD simulation trajectory by removing the overall rotation of the protein.

(3) The overall and internal motions are assumed to be independent and the overall rotational correlation function is calculated from eq 6 as $C_{\mathrm{O}}(t)=C(t) / C_{\mathrm{I}}(t)$.
(4) The mean square angle deviations of rotation around protein inertia axes are calculated from the MD simulation trajectory.

(5) rotational diffusion constants $D_{x}, D_{y}$, and $D_{z}$ around inertia axes are calculated by fitting a straight line to the mean square angle deviations

$$
\begin{aligned}
\left\langle\Delta \alpha_{t^{\prime}+t}{ }^{2}\right\rangle_{t^{\prime}} & =2 D_{x} t \\
\left\langle\Delta \beta_{t^{\prime}+t}{ }^{2}\right\rangle_{t^{\prime}} & =2 D_{y} t \\
\left\langle\Delta \gamma_{t^{\prime}+t}{ }^{2}\right\rangle_{t^{\prime}} & =2 D_{z} t
\end{aligned}
$$

where $\left\langle\Delta \alpha_{t^{\prime}+t}{ }^{2}\right\rangle_{t^{\prime}},\left\langle\Delta \beta_{t^{\prime}+t}{ }^{2}\right\rangle_{t^{\prime}}$, and $\left\langle\Delta \gamma_{t^{\prime}+t}{ }^{2}\right\rangle_{t^{\prime}}$ are the mean square angle deviations of the rotation around inertia axes from the longest protein inertia axis to the shortest, respectively.

(6) contribution of the overall rotational tumbling to all correlation functions is assumed to follow eq 8 with the timescales $\tau_{j}$ calculated from the rotational diffusion constants by using the relations in eq 9. Weighting factors $A_{j}$ are determined by fitting the equation to the overall rotational correlation functions calculated from MD simulations in step 3.

(7) The new correlation functions are calculated by substituting internal correlation functions, $C_{\mathrm{I}}(t)$, from step 2 and anisotropic rigid body rotational correlation functions, $C_{\mathrm{O}}(t)$, from step 6 to eq 6 giving

$$
C_{\mathrm{N}}(t)=C_{\mathrm{I}}(t) \sum_{j=1}^{5} A_{j} \mathrm{e}^{-t / \tau_{j}}
$$

These correlation functions are then used to calculate spin relaxation times from eqs $1-5$. The incorrect overall rotational diffusion due to a water model can be corrected at this point by scaling the rotational diffusion coefficients, that is, timescales $\tau_{j}$, with a constant factor before calculating new correlation functions from eq 13. Here, we determine the optimal scaling factors separately for each system. Scaling factors between 1 and 4 are explored with the spacing of 0.1 and the value giving the best agreement with the experimental spin relaxation data is selected to be the optimal scaling factor.

Simulation and Analysis Details. All simulations were performed using Gromacs $5^{54}$ software and Amber ff99SB$\mathrm{ILDN}^{55}$ force field for proteins. The protein was solvated to tip $3 p,^{56}$ tip $4 p,{ }^{56}$ or $\mathrm{OPC}^{57}$ water models. Initial structures were taken from the lowest-energy NMR structures of HpTonB-92 (PDB code: 5LW8) ${ }^{34}$ and PaTonB-96 (PDB code: $6 \mathrm{FIP}) .^{35}$ The results from different initial conformations of both proteins with the tip3p water model are shown in Section S2 in the Supporting Information. The temperature was coupled to the desired value with the v-rescale thermostat, ${ }^{58}$ and the pressure was isotropically set to 1 bar using a Parrinello-Rahman barostat. ${ }^{59}$ Time step was 2 fs, LennardJones interactions were cut off at $1.0 \mathrm{~nm}$, particle mesh 
Table 1. Simulated Systems and Rotational Diffusion Coefficients $\left(\operatorname{rad}^{2} \cdot 10^{7} / \mathrm{s}\right)$ Calculated from Simulations

$\begin{array}{clccccccccc}\text { protein } & \text { water } & T(\mathrm{~K})^{b} & t_{\mathrm{s}}(\mathrm{ns})^{c} & t_{\mathrm{a}}(\mathrm{ns})^{d} & D_{x} & D_{y} & D_{z} & D_{\|} / D_{\perp}{ }^{e} & D_{\mathrm{av}}{ }^{f} & \text { files }^{g} \\ \text { PaTonB-96 } & \text { tip3p } & 298 & 400 & 300 & 4.2 \pm 0.1 & 4.4 \pm 0.1 & 10.4 \pm 0.1 & 2.42 \pm 0.1 \\ \text { PaTonB-96 } & \text { tip4p } & 298 & 400 & 390 & 1.81 \pm 0.01 & 2.06 \pm 0.03 & 4.55 \pm 0.03 & 2.35 \pm 0.04 & 2.80 \pm 0.02 & 46 \\ \text { PaTonB-96 } & \text { tip4p } & 310 & 400 & 390 & 2.60 \pm 0.02 & 2.22 \pm 0.05 & 5.0 \pm 0.1 & 2.07 \pm 0.09 & 3.26 \pm 0.07 & 47 \\ \text { PaTonB-96 } & \text { OPC4 } & 310 & 1200 & 1190 & 2.01 \pm 0.01 & 2.19 \pm 0.01 & 5.01 \pm 0.03 & 2.39 \pm 0.02 & 3.07 \pm 0.01 & 48 \\ \text { HpTonB-92 } & \text { tip3p } & 310 & 570 & 370 & 8.25 \pm 0.05 & 7.67 \pm 0.06 & 15.9 \pm 0.3 & 1.99 \pm 0.06 & 10.6 \pm 0.2 & 49 \\ \text { HpTonB-92 } & \text { tip3p } & 303 & 800 & 790 & 6.24 \pm 0.02 & 7.04 \pm 0.03 & 11.9 \pm 0.2 & 1.80 \pm 0.03 & 8.40 \pm 0.07 & 50 \\ \text { HpTonB-92 } & \text { tip4p } & 310 & 470 & 370 & 3.6 \pm 0.1 & 3.24 \pm 0.01 & 6.3 \pm 0.3 & 1.8 \pm 0.1 & 4.4 \pm 0.2 & 51 \\ \text { HpTonB-92 } & \text { tip4p } & 303 & 400 & 200 & 2.7 \pm 0.1 & 2.71 \pm 0.02 & 5.6 \pm 0.5 & 2.1 \pm 0.2 & 3.7 \pm 0.2 & 52 \\ \text { HpTonB-92 } & \text { OPC4 } & 310 & 800 & 790 & 2.85 \pm 0.01 & 2.70 \pm 0.01 & 5.56 \pm 0.01 & 2.00 \pm 0.01 & 3.70 \pm 0.01 & 53\end{array}$

${ }^{a}$ Water model used in simulation. ${ }^{b}$ Simulation temperature. ${ }^{c}$ Total simulation time. ${ }^{d}$ Analyzed simulation time. ${ }^{e} D_{\|}=D_{z}, \quad D_{\perp}=\frac{1}{2}\left(D_{x}+D_{y}\right)$ $f_{D_{\mathrm{av}}}=\frac{1}{3}\left(D_{x}+D_{y}+D_{z}\right){ }^{g}$ Citation to a repository containing the simulation data.

Ewald $^{60,61}$ was used for electrostatics, and LINCS was used to constrain all bond lengths. ${ }^{62}$ The simulated systems are listed in Table 1 with the references giving access to the trajectories and the related simulation files. Equilibration of the trajectories was followed by monitoring the protein root-mean-square-deviation, inertia tensor eigenvalues, and rotation angles. Sufficient amount of data was omitted from the beginning of simulation trajectories to remove the significant fluctuations in these parameters. If such fluctuations were not observed, the first 10 ns of the trajectory was omitted as an equilibration period.

The rotational correlation functions are calculated with $g m x$ rotacf from Gromacs package. ${ }^{63}$ The overall rotation was removed for $C_{\mathrm{I}}(t)$ calculation by using a fit option of the gmx trjconv tool in Gromacs package. ${ }^{63}$ The order parameters $S^{2}$ were determined by averaging the rotational correlation functions from the oriented trajectory, $C_{\mathrm{I}}(t)$, over the lag times above $50 \mathrm{~ns}$. The effective correlation times were then calculated by eq 7 . Inertia axes of proteins were calculated with the compute_inertia_tensor function from MDTraj python library. ${ }^{6}$

Spectral density was calculated by fitting a sum of 471 exponentials with timescales from 1 ps to $50 \mathrm{~ns}$ with logarithmic spacing

$$
C_{\mathrm{N}}(t)=\sum_{i=1}^{N} \alpha_{i} \mathrm{e}^{-t / \tau_{i}}
$$

to the new correlation function from eq 13 by using the lsqnonneg routine in MATLAB. ${ }^{65}$ The Fourier transform was then calculated by using the analytical function for the sum of exponentials

$$
J(\omega)=2 \sum_{i=1}^{N} \alpha_{i} \frac{\tau_{i}}{1+\omega^{2} \tau_{i}^{2}}
$$

A similar approach has been previously used for the lamellar lipid and surfactant systems in combination with solid-state NMR experiments. ${ }^{66,67}$ All computer programs used for the analysis are available from ref 68.

Spin Relaxation Experiments. NMR experiments were recorded on a Bruker Avance III HD NMR spectrometer operated at ${ }^{1} \mathrm{H}$ frequency of $850.4 \mathrm{MHz}$ equipped with a cryogenic probe head. The longitudinal $\left(T_{1}\right)$, transverse $\left(T_{2}\right)$, and ${ }^{1} \mathrm{H}-{ }^{15} \mathrm{~N}$-heteronuclear NOE spin relaxation times for the backbone ${ }^{15} \mathrm{~N}$ atoms of $\mathrm{HpT}$ TonB-92 ${ }^{34}$ were collected at $303 \mathrm{~K}$ using the well-established NMR pulse sequences described previously. ${ }^{37,69,70}$ The similarly detected spin relaxation data for $\mathrm{PaTonB}-96$ at $298 \mathrm{~K}$ are also reported in another publication. ${ }^{35}$
The $T_{1}$ and $T_{2}$ relaxation times were measured using the following series of the delays: 10, 50, 100, 200, 300, 500, 800, 1000,1200 , and $2000 \mathrm{~ms}$ for $T_{1}$ and $16,64,96,128,156,196$, 224 , and $256 \mathrm{~ms}$ for $T_{2}$. Recycle delays of 3.0 and $2.0 \mathrm{~s}$ were used for $T_{1}$ and $T_{2}$ experiments, respectively. The relaxation rates $\left(R_{1}=1 / T_{1}, R_{2}=1 / T_{2}\right)$ were calculated as an exponential fit of a single exponential decay to peak intensity values: $I(t)=$ $I_{0} \exp \left(-t / T_{1}\right)$ or $I(t)=I_{0} \exp \left(-t / T_{2}\right)$, where $I(t)$ is the peak volume at a time $t$. The ${ }^{15} \mathrm{~N}\left\{{ }^{1} \mathrm{H}\right\}$-NOE measurements were carried out with a recycling delay of $5.1 \mathrm{~s}$ with and without saturation of the amide protons. The ${ }^{15} \mathrm{~N}\left\{{ }^{1} \mathrm{H}\right\}$-NOE values were derived from the volumes of the heteronuclear singlequantum coherence (HSQC) peaks using the equation of $\nu=$ $I / I_{0}$. The relaxation data were processed and analyzed using Bruker Dynamic Center software (version 2.1.8).

\section{RESULTS AND DISCUSSION}

Global Rotational Dynamics of the Protein. The mean square angle deviations for the rotation of the $\mathrm{PaTonB}-96$ protein around inertia axes in the simulation with the OPC4 water model are shown in Figure 1. This is the longest simulation data set in this work $(1.2 \mu \mathrm{s})$, and the linear behavior of the mean square angle deviations is observed for the lag times up to one hundredth of the total simulation length ( $12 \mathrm{~ns}$ ), which is expected to be the maximum lag time for the good statistics of rotational dynamics analyzed from a single molecule in MD simulations. ${ }^{33}$ Deviations from the linear behavior are only seen with the lag times longer than this limit, as also demonstrated for the shorter simulations with tip4p water at two different temperatures in Figures S1 and S2 in the Supporting Information. The plots with $\log -\log$ scale in Figures 1, S1, and S2 reveal a weakly subdiffusive region only below very short timescales of approximately $0.12 \mathrm{~ns}$. Thus, we conclude that the protein experiences the Brownian rotational tumbling with a good approximation. The diffusion coefficients can be then calculated from the slope of the mean square angle deviations according to eq 12 by using the lag times less than one hundredth of the total MD simulation length. The error bars were calculated by varying the lag time with $1 \mathrm{~ns}$ to both directions. The data from HpTonB-92 protein (not shown) led to similar conclusions.

The resulting rotational diffusion constants from different simulations are summarized in Table 1. As expected, the rotational diffusion coefficients increase with the temperature and the decreasing size of a protein. The values are, however, larger than expected from the experimental $T_{1} / T_{2}$ ratio analyzed with eq 11 and from the previously reported values 


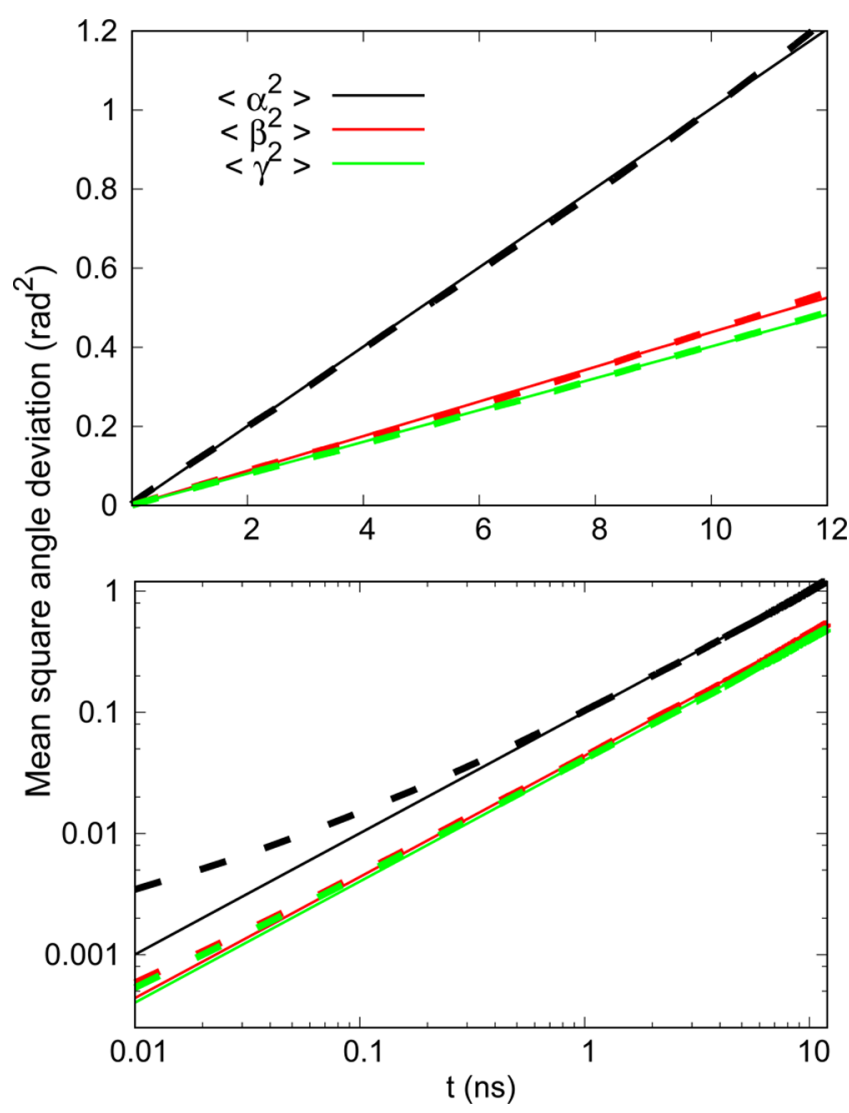

Figure 1. Mean square angle deviations of the rotation around inertia tensor axes calculated from $\mathrm{PaTonB}-96$ simulation with the OPC water model. The data are shown with linear (top) and logarithmic scale (bottom).

for proteins with similar sizes, ${ }^{71}$ especially when tip3p water model is used. Similar results were previously explained by the overestimated water self-diffusion of the tip3p water model. ${ }^{19,29,32}$

The analysis leading to the new correlation functions in eq 13 (see Methods section) is exemplified in Figure 2 for three residues located in different domains of $\mathrm{PaTonB}-96$ with different characteristic rotational dynamics. The flexible Cterminus is represented by the residue 341 , more rigid $\beta$-sheet by the residue 331 , and a flexible loop between two $\beta$-strands by residue 322 (see the labeling in Figure 6). The total correlation functions $C(t)$ of all residues in Figure 2 (top, solid lines) decay toward zero within $\sim 10-50$ ns. The internal correlation functions $C_{\mathrm{I}}(t)$ in Figure 2 (middle) decay to a plateau value, which defines the square of the order parameter $S^{2}$. As expected, the internal correlation function for residue 331 in the rigid $\beta$-sheet rapidly decays to the largest order parameter value, whereas the correlation functions of the residues in the loop and C-terminus decay slower to the smaller order parameter values because of the larger conformational ensemble sampled by these regions.

The overall rotational correlation functions, $C_{\mathrm{O}}(t)=C(t) /$ $C_{\mathrm{I}}(t)$, are shown in Figure 2 (bottom, solid lines). Also, the correlation functions of anisotropic rigid body rotation from eq 8 are shown in Figure 2 (bottom, dashed lines). The timescales for the latter, $\tau_{i}$, are given by the rotational diffusion coefficients from the simulation and the relations in eq 9. The prefactors, $A_{j}$, are determined by fitting eq 8 to the overall rotation correlation functions, $C_{\mathrm{O}}(t)$, calculated from the $\mathrm{MD}$

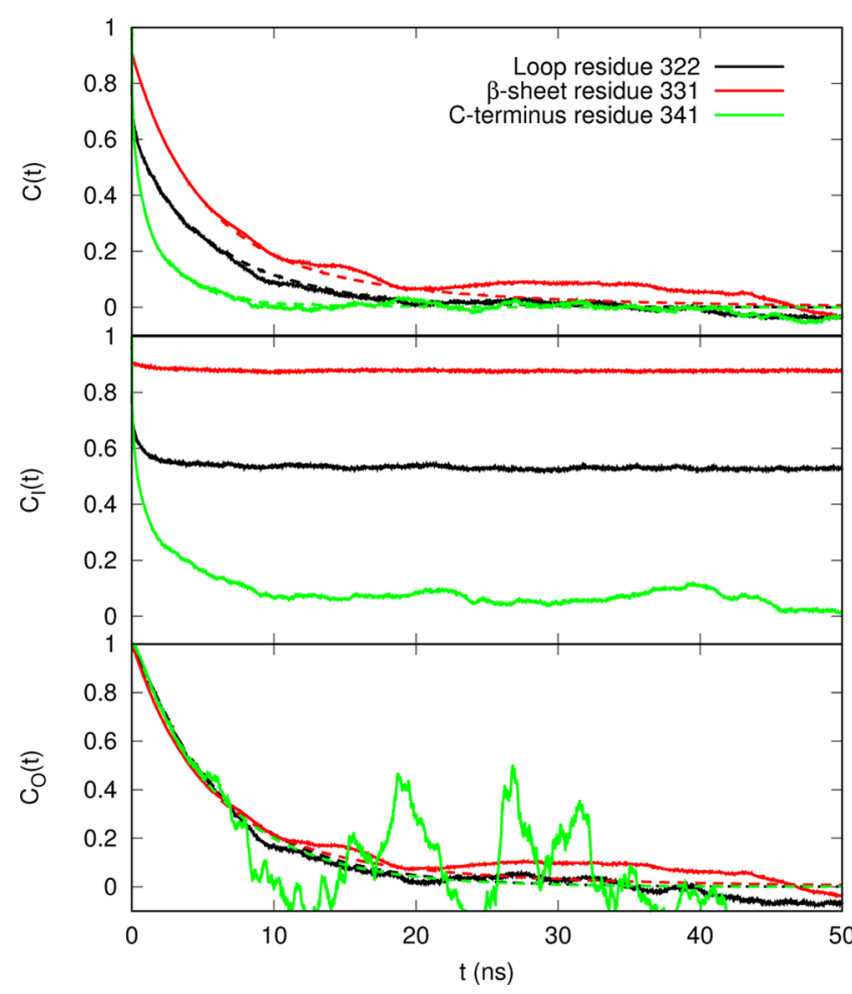

Figure 2. Rotational correlation functions calculated from $\mathrm{MD}$ simulations of PaTonB-96 with the tip4p water model at $298 \mathrm{~K}$ for residues at different regions. (Top) Total correlation functions $C(t)$ calculated from $\mathrm{MD}$ simulation (solid lines) and new correlation functions determined from eqs 6 and 8 by using rotational diffusion constants and fitted prefactors (dashed lines); (middle) correlation functions for internal motions calculated from simulation with removed overall protein rotation; and (bottom) correlation function for overall motions determined as $C_{\mathrm{O}}(t)=C(t) / C_{\mathrm{I}}(t)$ (solid lines) and by fitting to eq 8 with timescales from rotational diffusion coefficients in Table 1 (dashed lines).

simulation. The new correlation functions, determined from eq 13 and shown in Figure 2 (top, dashed lines), are indistinguishable from the correlation functions calculated from the original MD simulations with the lag times shorter than one hundredth of the total simulation time (approximately 4-12 ns), which is the maximum lag time for the good statistics in single-molecule MD simulations. ${ }^{33}$ This suggests that the anisotropic rigid body diffusion model (eq 8) and the separation of internal and global motions (eq 6) are good approximations for the proteins studied in this work. The analytical description of the overall rotation with eq 8 in the new correlation functions clearly reduces the statistical fluctuations with the long lag times in Figure 2. The effect is most visible for the flexible C-terminus (residue 341) having the smallest, thus the least detectable, contribution from the overall rotation of the protein due to the small order parameters.

Global Rotational Dynamics in Simulations and Experiments. Spin relaxation times of $\mathrm{HpTonB}-92$ are compared between the experiments and simulations using two different water models in Figure 3. The simulation with tip $3 p$ water model underestimates the $T_{1} / T_{2}$ ratios, suggesting too fast overall rotational diffusion dynamics. ${ }^{72}$ This is in agreement with the previous study, where the overestimated rotational diffusion was attributed to the self-diffusion of tip3p. ${ }^{19,29,32}$ On the other hand, simulation results with tip4p 

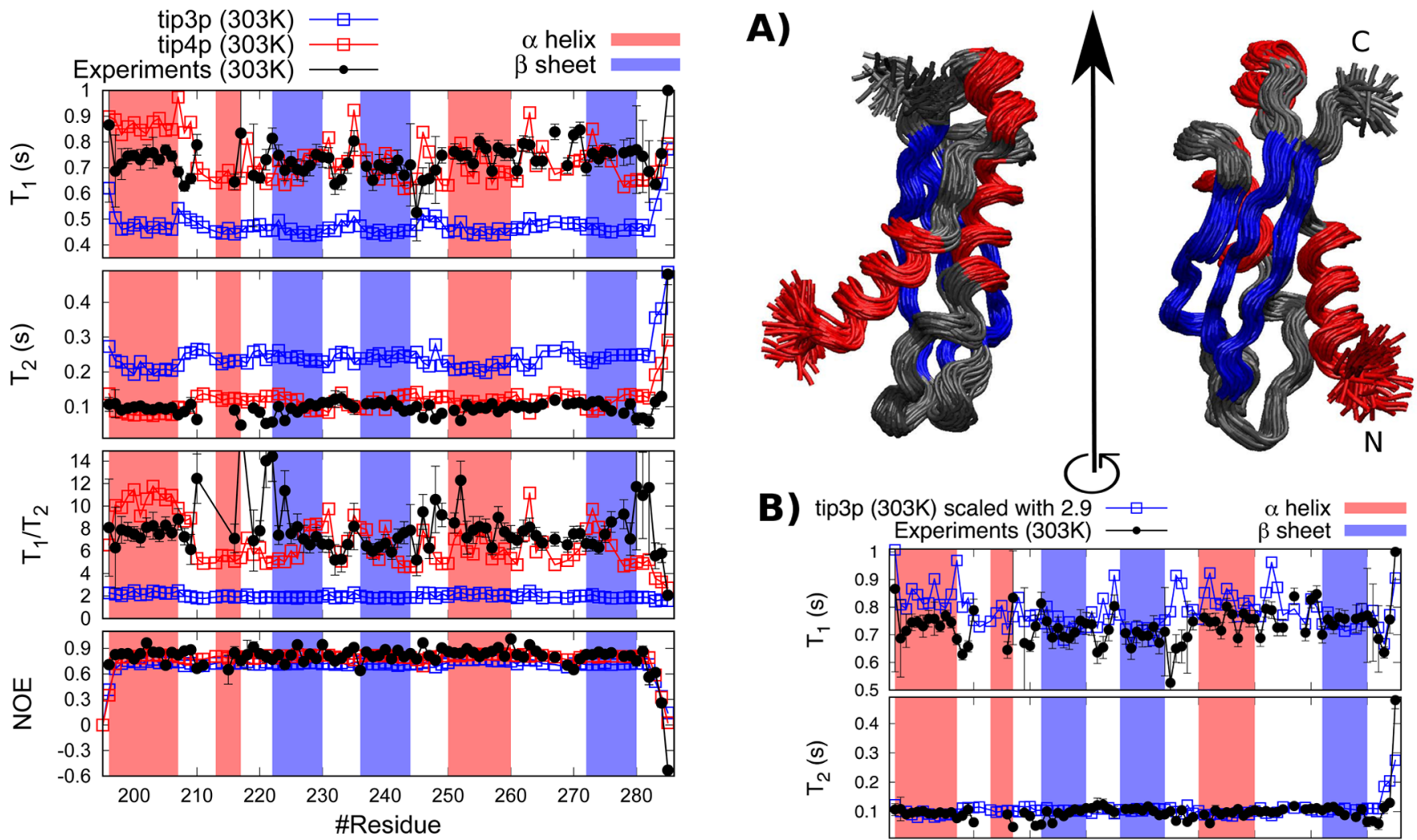

Figure $3 .{ }^{15} \mathrm{~N}$ spin relaxation times for $\mathrm{HpT}$ TonB-92 from experimental data (circles) and MD simulations with different water models (squares).

water model show better agreement with the experimental data in Figure 3.

To see if the discrepancy in spin relaxation times for simulations with tip3p water model could be explained by the overestimated overall diffusion of the protein, the diffusion coefficients were divided by the optimal scaling factor before applying eq 13 to calculate the new correlation functions. The scaling factor value of 2.9 gave the best agreement with the experimental spin relaxation data. The spin relaxation times calculated from the new correlation functions after scaling the rotational diffusion coefficients with the optimal scaling factor value are shown in Figure 4.

Similar comparison for the spin relaxation times of $\mathrm{PaTonB}$ 96 between experiments and simulations with tip3p, tip4p, and OPC4 water models is shown in Figure 5. The experimentation of the OPC4 water model was inspired by the recent study reporting significant improvements in lipid monolayer simulations when this water model was used. ${ }^{75}$ The underestimation of $T_{1} / T_{2}$ ratio was also observed in the simulations of $\mathrm{PaTonB}$ 96 with tip4p and OPC4 water models when compared with the experiments. The discrepancy is, however, less severe than with tip3p, suggesting that the required scaling factor for the overall rotational diffusion should be smaller for tip4p and OPC4 water models. Indeed, the spin relaxation times calculated from $P a$ TonB-96 simulation with the tip4p water model were found to be in good agreement with the experiments in Figure 6 when the diffusion coefficients were divided with a constant factor of 1.2, which is smaller than 2.9 used for the tip3p simulation of HpTonB-92 above. The scaling factors used to correct the overall rotational diffusion of different proteins with different water models are shown in

\section{B)}
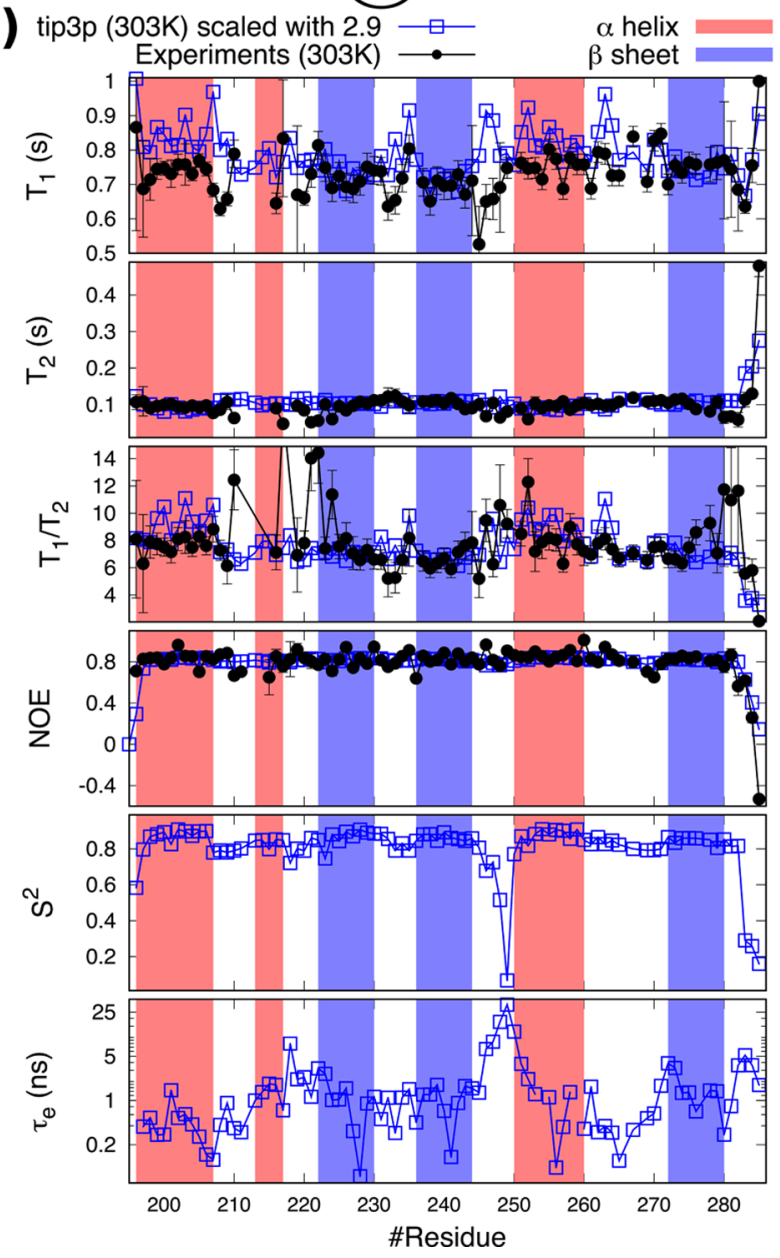

Figure 4. (A) Structures of $H p$ TonB-92 from the MD simulations with tip3p at $303 \mathrm{~K}$ (100 structures taken from $400 \mathrm{~ns}$ long trajectory). Secondary structures are color-labeled with Visual Molecular dynamics; ${ }^{73,74} \alpha$-helices are highlighted in red and $\beta$-strands in blue. Terminal ends are labeled with $\mathrm{N}$ and $\mathrm{C}$. The structure from left is rotated with approximately $150^{\circ}$ to the figure on right. (B) Spin relaxation times from experiments (circles) and tip $3 p$ simulations (squares) with rotational diffusion coefficients divided by a constant factor of 2.9 at $303 \mathrm{~K}$. Order parameters and effective internal correlation times calculated from simulations.

Table S1 together with the corresponding coefficients for selfdiffusion of water. ${ }^{29,57}$ Notably, the effect of $12{ }^{\circ} \mathrm{C}$ temperature difference on the spin relaxation times from tip4p simulations in Figure 5 is significantly smaller than the observed differences 


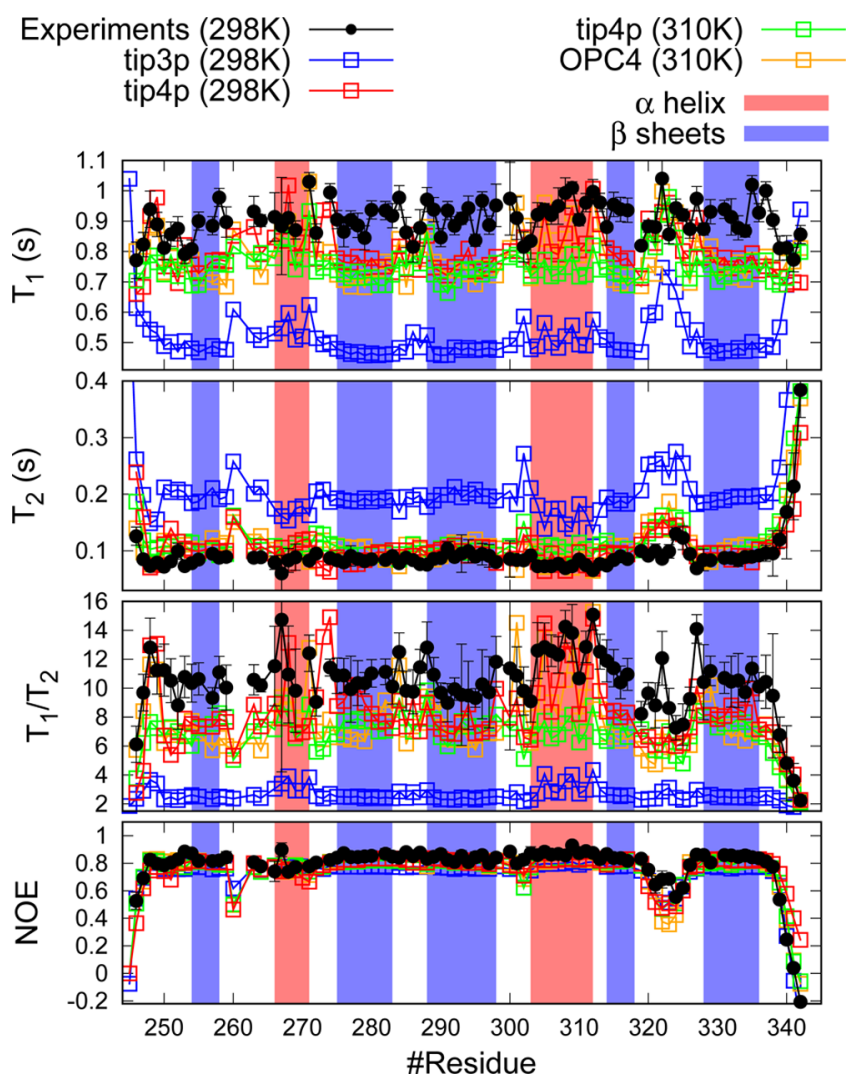

Figure 5. Plots of experimental (circles) and simulated (squares) spin relaxation times for $P a$ TonB-96.

between simulations and experiments or the changes due to the scaling of the diffusion coefficient.

The scaling of the overall rotational diffusion coefficients with a constant factor led to a good agreement with the experimental spin relaxation data for both systems simulated with different water models, as seen in Figures 4 and 6 . The good agreement with experiments suggests that the scaled rotational diffusion coefficients from $\mathrm{MD}$ simulations can be considered as an interpretation of the anisotropic rotational motion in NMR experiments. The scaled rotational diffusion coefficients from the simulations giving the best agreement with the experimental data are summarized in Table 2. In contrast to the unscaled diffusion constants in Table 1 , these results are in line with the previously reported values for proteins with similar sizes. ${ }^{71}$ Also, the timescales, $\tau_{c}^{\prime}$, estimated from eq 11 are close to the average diffusion coefficient, $\tau_{\mathrm{c}}=\left(6 D_{\mathrm{av}}\right)^{-1}$, in Table 1 .

Interpretation of Protein Internal Relaxation from MD Simulations. The good agreement of the spin relaxation times between the simulations with the scaled overall rotational diffusion coefficients and the experiments (Figures 4 and 6) suggests that the simulations can be used to interpret the internal mobility of proteins from the experimental data.

Only small variations between different residues are observed for spin relaxation times of $H p$ TonB-92 in Figure 4. This indicates a rather rigid protein structure, which is also seen in the MD simulation snapshots overlayed in Figure 4A. Only few residues in the terminal ends show slightly enhanced conformational fluctuations in the MD simulation and in spin relaxation data. In addition, some deviations from the average spin relaxation times are observed in the experimental data close to residues 210-222. Simulations of HpTonB-92 do not
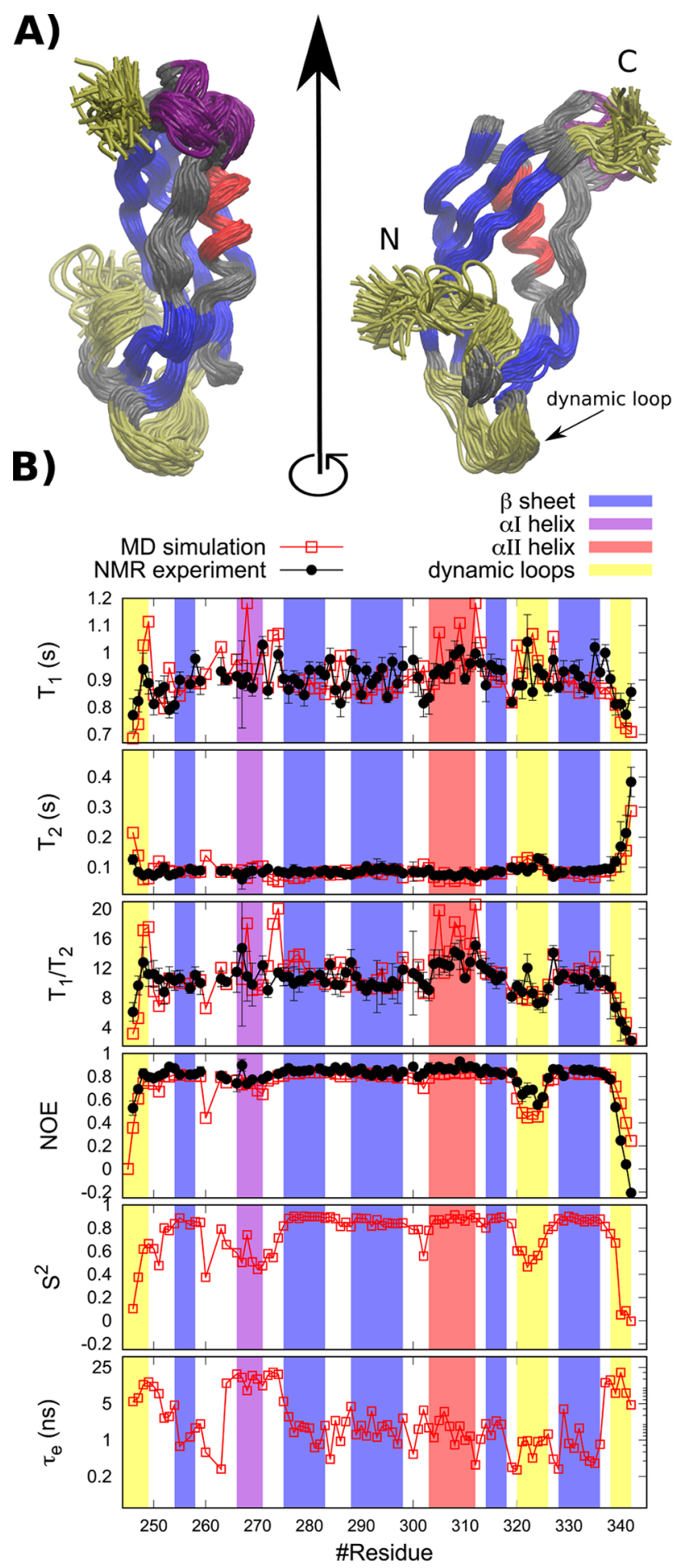

Figure 6. (A) Structures sampled by $P a T$ TonB-96 from $M D$ simulations with tip4p at $298 \mathrm{~K}$ (100 structures from $400 \mathrm{~ns}$ long trajectory). Secondary structures are color-labeled with Visual Molecular dynamics; ${ }^{73,74} \alpha$-helixes and $\beta$-strands are red and blue, respectively. Residues 246-251, 320-326, and 338-342 with increased internal dynamics are yellow and $\alpha$-helix fluctuations between two orientations (residues 266-270) are violet in the left column. Terminal ends are labeled with $\mathrm{N}$ and $\mathrm{C}$. The structure from left is rotated with approximately $100^{\circ}$ to the figure on right. (B) Spin relaxation times from experiments (circles) and tip $4 p$ simulations (squares) with rotational diffusion coefficients divided by a constant factor of 1.2 at 298 K. Order parameters and effective internal correlation times calculated from simulations. 
Table 2. Rotational Diffusion Coefficients $\left(\operatorname{rad}^{2} \cdot 10^{7} / \mathrm{s}\right)$ Giving the Best Agreement with Experimental Spin Relaxation data $^{a}$

$\begin{array}{lcc} & \text { HpTonB-92 } & \text { PaTonB-96 } \\ D_{x} & 2.15 \pm 0.01 & 1.51 \pm 0.01 \\ D_{y} & 2.43 \pm 0.01 & 1.72 \pm 0.03 \\ D_{z} & 4.10 \pm 0.01 & 3.79 \pm 0.03 \\ D_{\mathrm{av}} & 2.90 \pm 0.03 & 2.30 \pm 0.02 \\ \tau_{\mathrm{c}}(\mathrm{ns})^{b} & 5.7 \pm 0.1 & 7.2 \pm 0.1 \\ \tau_{\mathrm{c}}^{\prime}(\mathrm{ns})^{c} & 5.8 \pm 0.1 & 6.9 \pm 0.1\end{array}$

${ }^{a}$ For $H p$ TonB-92 construct, the values calculated from simulation with tip3p were scaled with 2.9 (spin relaxation data in Figure 4), and for $\mathrm{PaT}$ TonB-96, the values from tip4p simulation at $298 \mathrm{~K}$ were scaled by 1.2 (spin relaxation data in Figure 6). ${ }^{b} \tau_{\mathrm{c}}=\left(6 D_{\mathrm{av}}\right)^{-1}$. ${ }^{c}$ Average overall residues given by eq 11 .

offer any explanation for this observation; however, the similar region in $\mathrm{PaTonB}-96$ simulation shows fluctuations between two orientations of $\alpha$-helix. ${ }^{35}$ Exceptionally low order parameters and long effective correlation times are observed in simulations for residues 245-250 of $\mathrm{HpTonB}-92$. Moreover, short $T_{1}$ times are experimentally observed close to this region, but the interpretation is not straightforward as the low $T_{1}$ times are not reproduced by MD simulations.

$\mathrm{PaTonB}-96$ exhibits more internal mobility and the segments with enhanced conformational fluctuations are labeled with yellow color in Figure 6. The larger number of sampled conformations in both terminal ends is characterized by the low order parameters and long effective internal correlation times observed in the simulations. Enhanced conformational fluctuations are also observed for residues 320-326, which correspond to the loop between two $\beta$-strands. MD simulations predict low order parameters and long internal effective correlation times also close to residues 266-271, which can be explained by two different orientations sampled by the $\alpha$ helix in this region (color-labeled with violet in Figure 6A). The orientational fluctuations of the similar short helix could also explain the above mentioned deviations of spin relaxation times for residues $210-222$ of $\mathrm{HpT}$ TonB-92. ${ }^{34}$

MD simulations can be used to analyze different components contributing to the rotational dynamics of individual $\mathrm{N}-\mathrm{H}$ bonds. In this work, we have fitted a sum of 471 different timescales to the correlation functions according to eq 14. Most of the prefactors $\left(\alpha_{i}\right.$ in eq 14) are zero in all correlation functions after the fitting; thus, the timescales $\tau_{i}$ corresponding to nonzero prefactors are considered as the components contributing to the total relaxation process of each $\mathrm{N}-\mathrm{H}$ bond. The prefactors are shown in Figure 7 for the same residues of $P a$ TonB-96, which were used to exemplify the correlation functions in Figure 2. As expected for residue 322 in the rigid $\beta$ sheet with large order parameter, the rotational relaxation is dominated by timescales of $\sim 5.5$ and $\sim 8 \mathrm{~ns}$, matching with the protein overall rotation. Also, the dynamics of residue 322 in the flexible loop of $\mathrm{PaTonB}$ is dominated by the timescales around $\sim 8$ ns corresponding to the protein overall rotation; however, the fast motions from internal mobility are more evident than for the rigid $\beta$-sheet residue. This is in agreement with smaller order parameter observed in the flexible loop residues. On the other hand, the rotational dynamics of residue 341 in the flexible N-terminus of $P a T$ TonB is dominated by timescales below $3 \mathrm{~ns}$, most likely related to the internal motion of the protein. Contributions from timescales around $\sim 13 \mathrm{~ns}$ to

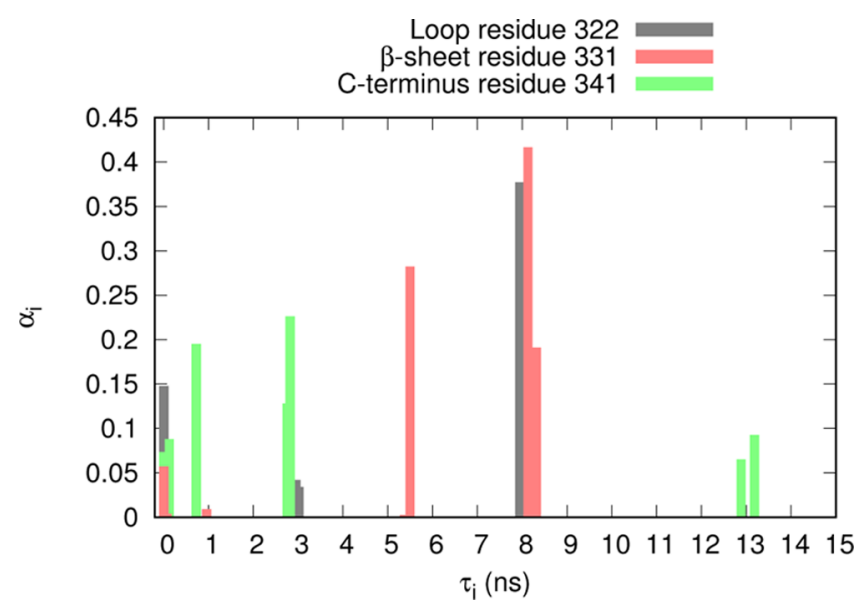

Figure 7. Prefactors $\alpha_{i}$ corresponding to different timescales $\tau_{i}$ resulting from a fit of eq 14 to correlation functions from MD simulation of $\mathrm{PaT}$ TonB-96 at $298 \mathrm{~K}$. The used correlation functions give a good agreement with experimental spin relaxation times as shown in Figure 6.

the dynamics of residue 341 probably arise from the slow conformational fluctuations of the $\mathrm{N}$-terminus, rather than the overall rotational dynamics. This supports the conclusion that the large amount of sampled conformations lead to the small order parameters and large effective correlation times observed in Figure 5. Although the separation of rotational dynamics of individual $\mathrm{N}-\mathrm{H}$ bonds to different components gives intuitively understandable results, it should be kept in mind that it is based on the fitting of a multiexponential sum to the simulation data and the solution of such fit is not unique.

\section{CONCLUSIONS}

The experimental spin relaxation data for protein backbone N$\mathrm{H}$ bonds were successfully reproduced by using the classical $\mathrm{MD}$ simulations for two different small domains. Thus, the simulation trajectories give an atomic resolution interpretation for protein dynamics measured with NMR experiments. Interpretation of the overall and internal dynamics was demonstrated for two proteins with anisotropic molecular shape and some flexible regions. Interpretation of the ${ }^{15} \mathrm{~N}$ spin relaxation data measured from such proteins has been very challenging with the previously available methods. ${ }^{26,69}$

The overall rotation of the studied proteins was found to be Brownian, having only a small subdiffusive behavior with short timescales below $\sim 0.12$ ns, which could be contrasted with crowded environments, where anomalous diffusion is expected to be more significant. ${ }^{76}$ The direct analysis of classical MD trajectories did not, however, reproduce the experimental ${ }^{15} \mathrm{~N}$ spin relaxation data. Comparison between the rotational diffusion coefficients and spin relaxation times between simulations and experiments suggested that the overall Brownian tumbling of proteins is too rapid in the simulations, in agreement with the previous report suggesting that the discrepancy arises from the inaccuracies in water models. ${ }^{19,29,32}$ Scaling down the anisotropic diffusion coefficients in the simulation data led to a good agreement with the experimental data. Overall rotational diffusion coefficients were overestimated by a factor of $\sim 3$ in the HpTonB-92 simulations with the tip3p water model, in line with previous studies. $^{28-30,77}$ Simulations with tip4p and OPC4 water models gave the spin relaxation times in reasonable agreement with 
experiments with scaling factors of $\sim 1-1.2$, which are significantly less than that for tip3p. The scaling factors for different proteins with different water models are summarized in Table S1.

The similarity between the correlation functions from the original $\mathrm{MD}$ trajectory and the new correlation functions from eq 13 suggests that the usage of the inertia axes and the separation of internal and the overall rotational motions (eq 6) are good approximations for the above investigated proteins. This is in line with the previous studies of other proteins with well-defined structure. ${ }^{10,29}$ However, it remains to be seen how well this and other related approaches ${ }^{28,30,78}$ will succeed for intrinsically disordered proteins without the well-defined shape. Because the correction of the incorrect overall rotational diffusion due to the water model may become highly complicated for such proteins, it may be necessary to employ a water model giving correct overall rotational diffusion coefficients for biomolecules. ${ }^{19,32}$

As further demonstrated in ref 35, the approach presented in this work can be used to interpret the rotational dynamics of proteins with anisotropic shape from ${ }^{15} \mathrm{~N}$ spin relaxation data measured only with one magnetic field strength. This is a significant advancement over currently available methods, which may not be applicable in such cases, even though experimental data would be measured with multiple magnetic field strengths.

\section{ASSOCIATED CONTENT}

\section{S Supporting Information}

The Supporting Information is available free of charge on the ACS Publications website at DOI: 10.1021/acs.jpcb.8b02250.

Mean square angle deviations of $\mathrm{PaTonB}-96$ simulations with tip4p water model at $310 \mathrm{~K}$ and $298 \mathrm{~K}$ and accuracy estimation of the scaling factors for the rotational diffusion (PDF)

\section{AUTHOR INFORMATION}

\section{Corresponding Author}

*E-mail: samuli.ollila@helsinki.fi.

\section{ORCID ${ }^{\circ}$}

O. H. Samuli Ollila: 0000-0002-8728-1006

Hideo Iwaï: 0000-0001-7376-5264

\section{Notes}

The authors declare no competing financial interest.

\section{ACKNOWLEDGMENTS}

Academy of Finland (277335) and Sigrid Jusélius Foundation are acknowledged for the financial support to complete this work. The Finnish Biological NMR Center is supported by Biocenter Finland and HiLIFE-INFRA. We acknowledge CSCIT center for science for computational resources.

\section{REFERENCES}

(1) Jarymowycz, V. A.; Stone, M. J. Fast Time Scale Dynamics of Protein Backbones: NMR Relaxation Methods, Applications, and Functional Consequences. Chem. Rev. 2006, 106, 1624-1671.

(2) Korzhnev, D. M.; Billeter, M.; Arseniev, A. S.; Orekhov, V. Y. NMR Studies of Brownian Tumbling and Internal Motions in Proteins. Prog. Nucl. Magn. Reson. Spectrosc. 2001, 38, 197-266.

(3) Mulder, F. A. A.; Mittermaier, A.; Hon, B.; Dahlquist, F. W.; Kay, L. E. Studying Excited States of Proteins by NMR Spectroscopy. Nat. Struct. Mol. Biol. 2001, 8, 932-935.
(4) Eisenmesser, E. Z.; Millet, O.; Labeikovsky, W.; Korzhnev, D. M.; Wolf-Watz, M.; Bosco, D. A.; Skalicky, J. J.; Kay, L. E.; Kern, D. Intrinsic Dynamics of an Enzyme Underlies Catalysis. Nature 2005, 438, 117-121.

(5) Van den Bedem, H.; Fraser, J. S. Integrative, Dynamic Structural Biology at Atomic Resolution-It's About Time. Nat. Methods 2015, $12,307-318$

(6) Lewandowski, J. R.; Halse, M. E.; Blackledge, M.; Emsley, L. Direct Observation of Hierarchical Protein Dynamics. Science 2015, 348, 578-581.

(7) Lamley, J. M.; Lougher, M. J.; Sass, H. J.; Rogowski, M.; Grzesiek, S.; Lewandowski, J. R. Unraveling the Complexity of Protein Backbone Dynamics with Combined 13C and 15N Solid-state NMR Relaxation Measurements. Phys. Chem. Chem. Phys. 2015, 17, 21997-22008.

(8) Yang, D.; Kay, L. E. Contributions to Conformational Entropy Arising from Bond Vector Fluctuations Measured from NMR-Derived Order Parameters: Application to Protein Folding. J. Mol. Biol. 1996, 263, 369-382.

(9) Kasinath, V.; Sharp, K. A.; Wand, A. J. Microscopic Insights into the NMR Relaxation-Based Protein Conformational Entropy Meter. J. Am. Chem. Soc. 2013, 135, 15092-15100.

(10) Allnér, O.; Foloppe, N.; Nilsson, L. Motions and Entropies in Proteins as Seen in NMR Relaxation Experiments and Molecular Dynamics Simulations. J. Phys. Chem. B 2015, 119, 1114-1128.

(11) Solomentsev, G.; Diehl, C.; Akke, M. Conformational Entropy of FK506 Binding to FKBP12 Determined by Nuclear Magnetic Resonance Relaxation and Molecular Dynamics Simulations. Biochemistry 2018, 57, 1451-1461.

(12) Akke, M.; Brueschweiler, R.; Palmer, A. G. NMR Order Parameters and Free Energy: an Analytical Approach and Its Application to Cooperative Calcium(2+) Binding by Calbindin D9k. J. Am. Chem. Soc. 1993, 115, 9832-9833.

(13) Sanchez-Medina, C.; Sekhar, A.; Vallurupalli, P.; Cerminara, M.; Muñoz, V.; Kay, L. E. Probing the Free Energy Landscape of the FastFolding gpW Protein by Relaxation Dispersion NMR. J. Am. Chem. Soc. 2014, 136, 7444-7451.

(14) Best, R. B.; Vendruscolo, M. Determination of Protein Structures Consistent with NMR Order Parameters. J. Am. Chem. Soc. 2004, 126, 8090-8091.

(15) Showalter, S. A.; Brüschweiler, R. Validation of Molecular Dynamics Simulations of Biomolecules Using NMR Spin Relaxation as Benchmarks: Application to the AMBER99SB Force Field. J. Chem. Theory Comput. 2007, 3, 961-975.

(16) Showalter, S. A.; Johnson, E.; Rance, M.; Brüschweiler, R. Toward Quantitative Interpretation of Methyl Side-Chain Dynamics from NMR by Molecular Dynamics Simulations. J. Am. Chem. Soc. 2007, 129, 14146-14147.

(17) Maragakis, P.; Lindorff-Larsen, K.; Eastwood, M. P.; Dror, R O.; Klepeis, J. L.; Arkin, I. T.; Jensen, M. Ø.; Xu, H.; Trbovic, N.; Friesner, R. A.; et al. Microsecond Molecular Dynamics Simulation Shows Effect of Slow Loop Dynamics on Backbone Amide Order Parameters of Proteins. J. Phys. Chem. B 2008, 112, 6155-6158.

(18) Trbovic, N.; Kim, B.; Friesner, R. A.; Palmer, A. G. Structural Analysis of Protein Dynamics by MD Simulations and NMR Spinrelaxation. Proteins: Struct., Funct., Bioinf. 2008, 71, 684-694.

(19) Debiec, K. T.; Cerutti, D. S.; Baker, L. R.; Gronenborn, A. M.; Case, D. A.; Chong, L. T. Further along the Road Less Traveled: AMBER ff15ipq, an Original Protein Force Field Built on a SelfConsistent Physical Model. J. Chem. Theory Comput. 2016, 12, 39263947.

(20) Hoffmann, F.; Mulder, F. A. A.; Schäfer, L. V. Accurate Methyl Group Dynamics in Protein Simulations with AMBER Force Fields. J. Phys. Chem. B 2018, 122, 5038-5048, DOI: 10.1021/ acs.jpcb.8b02769.

(21) Debiec, K. T.; Whitley, M. J.; Koharudin, L. M. I.; Chong, L. T.; Gronenborn, A. M. Integrating NMR, SAXS, and Atomistic Simulations: Structure and Dynamics of a Two-Domain Protein. Biophys. J. 2018, 114, 839-855. 
(22) Wennerstroem, H.; Lindman, B.; Soederman, O.; Drakenberg, T.; Rosenholm, J. B. Carbon-13 Magnetic Relaxation in Micellar Solutions. Influence of Aggregate Motion on T1. J. Am. Chem. Soc. 1979, 101, 6860-6864.

(23) Lipari, G.; Szabo, A. Model-Free Approach to the Interpretation of Nuclear Magnetic Resonance Relaxation in Macromolecules. 1. Theory and Range of Validity. J. Am. Chem. Soc. 1982, 104, 45464559.

(24) Woessner, D. E. Nuclear Spin Relaxation in Ellipsoids Undergoing Rotational Brownian Motion. J. Chem. Phys. 1962, 37, $647-654$.

(25) Shimizu, H. Effect of Molecular Shape on Nuclear Magnetic Relaxation. J. Chem. Phys. 1962, 37, 765-778.

(26) Luginbühl, P.; Pervushin, K. V.; Iwai, H.; Wüthrich, K. Anisotropic Molecular Rotational Diffusion in 15N Spin Relaxation Studies of Protein Mobility. Biochemistry 1997, 36, 7305-7312.

(27) Blake-Hall, J.; Walker, O.; Fushman, D. In Protein NMR Techniques; Downing, A. K., Ed.; Humana Press: Totowa, NJ, 2004; pp 139-159.

(28) Prompers, J. J.; Brüschweiler, R. General Framework for Studying the Dynamics of Folded and Nonfolded Proteins by NMR Relaxation Spectroscopy and MD Simulation. J. Am. Chem. Soc. 2002, 124, 4522-4534.

(29) Wong, V.; Case, D. A. Evaluating Rotational Diffusion from Protein MD Simulations. J. Phys. Chem. B 2008, 112, 6013-6024.

(30) Anderson, J. S.; LeMaster, D. M. Rotational Velocity Rescaling of Molecular Dynamics Trajectories for Direct Prediction of Protein NMR Relaxation. Biophys. Chem. 2012, 168, 28-39.

(31) Salvi, N.; Abyzov, A.; Blackledge, M. Multi-Timescale Dynamics in Intrinsically Disordered Proteins from NMR Relaxation and Molecular Simulation. J. Phys. Chem. Lett. 2016, 7, 2483-2489.

(32) Takemura, K.; Kitao, A. Water Model Tuning for Improved Reproduction of Rotational Diffusion and NMR Spectral Density. J. Phys. Chem. B 2012, 116, 6279-6287.

(33) Lu, C.-Y.; Bout, D. A. V. Effect of Finite Trajectory Length on the Correlation Function Analysis of Single Molecule Data. J. Chem. Phys. 2006, 125, 124701.

(34) Ciragan, A.; Aranko, A. S.; Tascon, I.; Iwaï, H. Salt-inducible Protein Splicing in cis and trans by Inteins from Extremely Halophilic Archaea as a Novel Protein-Engineering Tool. J. Mol. Biol. 2016, 428, $4573-4588$.

(35) Oeemig, J. S.; Ollila, O. H. S.; Iwaï, H. The NMR structure of the C-terminal domain of TonB protein from Pseudomonas aeruginosa, 2018, submitted.

(36) Abragam, A. The Principles of Nuclear Magnetism; Oxford University Press, 1961.

(37) Kay, L. E.; Torchia, D. A.; Bax, A. Backbone Dynamics of Proteins as Studied by Nitrogen-15 Inverse Detected Heteronuclear NMR Spectroscopy: Application to Staphylococcal Nuclease. Biochemistry 1989, 28, 8972-8979.

(38) Hiyama, Y.; Niu, C. H.; Silverton, J. V.; Bavoso, A.; Torchia, D. A. Determination of $15 \mathrm{~N}$ Chemical Shift Tensor via $15 \mathrm{~N}-2 \mathrm{H}$ Dipolar Coupling in Boc-glycylglycyl[15N glycine $]$ benzyl ester. J. Am. Chem. Soc. 1988, 110, 2378-2383.

(39) Halle, B. The Physical Basis of Model-free Analysis of NMR Relaxation Data From Proteins and Complex Fluids. J. Chem. Phys. 2009, 131, 224507.

(40) Dosset, P.; Hus, J.-C.; Blackledge, M.; Marion, D. Efficient Analysis of Macromolecular Rotational Diffusion from Heteronuclear Relaxation Data. J. Biomol. NMR 2000, 16, 23-28.

(41) de la Torre, J. G.; Huertas, M.; Carrasco, B. HYDRONMR: Prediction of NMR Relaxation of Globular Proteins from AtomicLevel Structures and Hydrodynamic Calculations. J. Magn. Reson. 2000, 147, 138-146.

(42) Fisette, O.; Lagüe, P.; Gagné, S.; Morin, S. Synergistic Applications of MD and NMR for the Study of Biological Systems. J. Biomed. Biotechnol. 2012, 2012, 254208.

(43) Anderson, J. S.; Hernández, G.; LeMaster, D. M. Prediction of Bond Vector Autocorrelation Functions from Larmor Frequency-
Selective Order Parameter Analysis of NMR Relaxation Data. J. Chem. Theory Comput. 2017, 13, 3276-3289.

(44) Gu, Y.; Li, D.-W.; Brüschweiler, R. NMR Order Parameter Determination from Long Molecular Dynamics Trajectories for Objective Comparison with Experiment. J. Chem. Theory Comput. 2014, 10, 2599-2607.

(45) Ollila, O. H. S. MD simulation data for Pseudomonas aeruginosa TonB-CTD. Amber ff99SB-ILDN, tip3p, 298K, Gromacs, 2018, http:// dx.doi.org/10.5281/zenodo.1244108.

(46) Ollila, O. H. S. MD simulation data for Pseudomonas aeruginosa TonB-CTD. Amber ff99SB-ILDN, tip4p, 298K, Gromacs, 2017, https:// doi.org/10.5281/zenodo.1010415.

(47) Ollila, O. H. S. MD simulation data for Pseudomonas aeruginosa TonB-CTD. Amber ff99SB-ILDN, tip4p, 310K, Gromacs, 2017, https:// doi.org/10.5281/zenodo.1010405.

(48) Ollila, O. H. S. MD simulation data for Pseudomonas aeruginosa TonB-CTD. Amber ff99SB-ILDN, OPC4, 310K, Gromacs, 2017, https://doi.org/10.5281/zenodo.1010437.

(49) Ollila, O. H. S. MD simulation data for Helicobacter pylori TonBCTD (residues 194-285) (PDB ID: 5LW8; BMRB entry: 34043). Amber ff99SB-ILDN, tip3p, 310K, Gromacs, 2017, https://doi.org/10. 5281/zenodo.1010231.

(50) Ollila, O. H. S. MD simulation data for Helicobacter pylori TonBCTD (residues 194-285) (PDB ID: 5LW8; BMRB entry: 34043). Amber ff99SB-ILDN, tip3p, 303K, Gromacs, 2017, https://doi.org/10. 5281/zenodo.1010141.

(51) Ollila, O. H. S. MD simulation data for Helicobacter pylori TonBCTD (residues 194-285) (PDB ID: 5LW8; BMRB entry: 34043). Amber ff99SB-ILDN, tip4p, 310K, Gromacs, 2017, https://doi.org/10. 5281/zenodo.1010237.

(52) Ollila, O. H. S. MD simulation data for Helicobacter pylori TonBCTD (residues 194-285) (PDB ID: 5LW8; BMRB entry: 34043). Amber ff99SB-ILDN, tip4p, 303K, Gromacs, 2017, https://doi.org/10. 5281/zenodo.1010351.

(53) Ollila, O. H. S.. MD simulation data for Helicobacter pylori TonB-CTD (residues 194-285) (PDB ID: 5LW8; BMRB entry: 34043). Amber ff99SB-ILDN, OPC4, 310K, Gromacs, 2017, https://doi. org/10.5281/zenodo.1010356.

(54) Abraham, M. J.; Murtola, T.; Schulz, R.; Páll, S.; Smith, J. C.; Hess, B.; Lindahl, E. GROMACS: High Performance Molecular Simulations Through Multi-level Parallelism from Laptops to Supercomputers. SoftwareX 2015, 1-2, 19-25.

(55) Lindorff-Larsen, K.; Piana, S.; Palmo, K.; Maragakis, P.; Klepeis, J. L.; Dror, R. O.; Shaw, D. E. Improved Side-chain Torsion Potentials for the Amber ff99SB Protein Force Field. Proteins: Struct., Funct., Bioinf. 2010, 78, 1950-1958.

(56) Jorgensen, W. L.; Chandrasekhar, J.; Madura, J. D.; Impey, R. W.; Klein, M. L. Comparison of Simple Potential Functions for Simulating Liquid Water. J. Chem. Phys. 1983, 79, 926-935.

(57) Izadi, S.; Anandakrishnan, R.; Onufriev, A. V. Building Water Models: A Different Approach. J. Phys. Chem. Lett. 2014, 5, 38633871.

(58) Bussi, G.; Donadio, D.; Parrinello, M. Canonical Sampling Through Velocity Rescaling. J. Chem. Phys. 2007, 126, 014101.

(59) Parrinello, M.; Rahman, A. Polymorphic Transitions in Single Crystals: A New Molecular Dynamics Method. J. Appl. Phys. 1981, 52, $7182-7190$.

(60) Darden, T.; York, D.; Pedersen, L. Particle Mesh Ewald: An N. $\log (\mathrm{N})$ Method for Ewald Sums in Large Systems. J. Chem. Phys. 1993, 98, 10089-10092.

(61) Essmann, U.; Perera, L.; Berkowitz, M. L.; Darden, T.; Lee, H.; Pedersen, L. G. A Smooth Particle Mesh Ewald Potential. J. Chem. Phys. 1995, 103, 8577-8593.

(62) Hess, B. P-LINCS: A Parallel Linear Constraint Solver for Molecular Simulation. J. Chem. Theory Comput. 2008, 4, 116-122.

(63) Abraham, M.; van der Spoel, D.; Lindahl, E.; Hess, B. The GROMACS development team, GROMACS user manual. Version 5.0.7, 2015 . 
(64) McGibbon, R. T.; Beauchamp, K. A.; Harrigan, M. P.; Klein, C.; Swails, J. M.; Hernández, C. X.; Schwantes, C. R.; Wang, L.-P.; Lane, T. J.; Pande, V. S. MDTraj: A Modern Open Library for the Analysis of Molecular Dynamics Trajectories. Biophys. J. 2015, 109, 15281532.

(65) MATLAB, R2016a; The MathWorks, Inc., Natick, Massachusetts, United States.

(66) Nowacka, A.; Bongartz, N. A.; Ollila, O. H. S.; Nylander, T.; Topgaard, D. Signal Intensities in $1 \mathrm{H}-13 \mathrm{C} \mathrm{CP}$ and INEPT MAS NMR of Liquid Crystals. J. Magn. Reson. 2013, 230, 165-175.

(67) Ferreira, T. M.; Ollila, O. H. S.; Pigliapochi, R.; Dabkowska, A. P.; Topgaard, D. Model-free Estimation of the Effective Correlation Time for C-H Bond Reorientation in Amphiphilic Bilayers: 1H-13C Solid-state NMR and MD Simulations. J. Chem. Phys. 2015, 142, 044905.

(68) Ollila, O. H. S. ProteinDynamics, 2017, https://doi.org/10. 5281/zenodo.1288573.

(69) Barbato, G.; Ikura, M.; Kay, L. E.; Pastor, R. W.; Bax, A. Backbone Dynamics of Calmodulin Studied by Nitrogen-15 Relaxation Using Inverse Detected Two-dimensional NMR Spectroscopy: The Central Helix is Flexible. Biochemistry 1992, 31, 5269-5278.

(70) Farrow, N. A.; Muhandiram, R.; Singer, A. U.; Pascal, S. M.; Kay, C. M.; Gish, G.; Shoelson, S. E.; Pawson, T.; Forman-Kay, J. D.; Kay, L. E. Backbone Dynamics of a Free and a PhosphopeptideComplexed Src Homology 2 Domain Studied by $15 \mathrm{~N}$ NMR Relaxation. Biochemistry 1994, 33, 5984-6003.

(71) Krishnan, V. V.; Cosman, M. An Empirical Relationship Between Rotational Correlation Time and Solvent Accessible Surface Area. J. Biomol. NMR 1998, 12, 177-182.

(72) Carper, W. R.; Keller, C. E. Direct Determination of NMR Correlation Times from Spin-Lattice and Spin-Spin Relaxation Times. J. Phys. Chem. A 1997, 101, 3246-3250.

(73) Frishman, D.; Argos, P. Knowledge-based Protein Secondary Structure Assignment. Proteins: Struct., Funct., Bioinf. 1995, 23, 566579.

(74) Humphrey, W.; Dalke, A.; Schulten, K. VMD - Visual Molecular Dynamics. J. Mol. Graphics 1996, 14, 33-38.

(75) Javanainen, M.; Lamberg, A.; Cwiklik, L.; Vattulainen, I.; Ollila, O. H. S. Atomistic Model for Nearly Quantitative Simulations of Langmuir Monolayers. Langmuir 2018, 34, 2565-2572.

(76) Höfling, F.; Franosch, T. Anomalous Transport in the Crowded World of Biological Cells. Rep. Prog. Phys. 2013, 76, 046602.

(77) Linke, M.; Köfinger, J.; Hummer, G. Fully Anisotropic Rotational Diffusion Tensor from Molecular Dynamics Simulations. J. Phys. Chem. B 2018, 122, 5630.

(78) Chen, P.-c.; Hologne, M.; Walker, O.; Hennig, J. Ab Initio Prediction of NMR Spin Relaxation Parameters from Molecular Dynamics Simulations. J. Chem. Theory Comput. 2018, 14, 1009-1019. 